ESAIM: M2AN 46 (2012) 1467-1483

DOI: $10.1051 / \mathrm{m} 2 \mathrm{an} / 2012010$
ESAIM: Mathematical Modelling and Numerical Analysis

www.esaim-m2an.org

\title{
DISCONTINUOUS GALERKIN METHODS FOR PROBLEMS WITH DIRAC DELTA SOURCE*
}

\author{
Paul Houston $^{1}$ and Thomas Pascal Wihler ${ }^{2}$
}

\begin{abstract}
In this article we study discontinuous Galerkin finite element discretizations of linear second-order elliptic partial differential equations with Dirac delta right-hand side. In particular, assuming that the underlying computational mesh is quasi-uniform, we derive an a priori bound on the error measured in terms of the $L^{2}$-norm. Additionally, we develop residual-based a posteriori error estimators that can be used within an adaptive mesh refinement framework. Numerical examples for the symmetric interior penalty scheme are presented which confirm the theoretical results.
\end{abstract}

Mathematics Subject Classification. 65N30.

Received June 17, 2011. Revised November 22, 2011.

Published online May 31, 2012.

\section{INTRODUCTION}

In this article, we will consider the numerical approximation of the boundary value model problem

$$
\begin{aligned}
-\Delta u & =\delta_{x_{0}} & & \text { in } \Omega, \\
u & =0 & & \text { on } \partial \Omega,
\end{aligned}
$$

based on employing discontinuous Galerkin (DG) finite element discretizations. Here, $\Omega \subset \mathbb{R}^{2}$ is an open bounded polygonal domain, and $\delta_{\boldsymbol{x}_{0}}$ denotes the Dirac delta distribution at some given point $\boldsymbol{x}_{0} \in \Omega$. Throughout, in order to avoid technical difficulties due to corner singularities, we suppose that the domain $\Omega$ is convex (this assumption can be relaxed in some parts of the article; this will be remarked on later). The weak formulation of (1.1)-(1.2) is to find $u \in W_{0}^{1, p}(\Omega)$ such that

$$
a(u, v):=\int_{\Omega} \nabla u \cdot \nabla v \mathrm{~d} \boldsymbol{x}=v\left(\boldsymbol{x}_{0}\right) \quad \forall v \in W_{0}^{1, q}(\Omega),
$$

Keywords and phrases. Elliptic PDEs, discontinuous Galerkin methods, Dirac delta source.

* Thomas Wihler acknowledges the financial support of the Swiss National Science Foundation (SNF) under Grant No. 200021_126594.

1 School of Mathematical Sciences, University of Nottingham, University Park, Nottingham, NG7 2RD, UK.

Paul.Houston@nottingham.ac.uk

2 Mathematics Institute, University of Bern, 3012 Bern, Switzerland. wihler@math.unibe.ch 
with $1 \leq p<2$, and $\frac{1}{p}+\frac{1}{q}=1$. In this manuscript, for $s \in \mathbb{N}_{0}$ and $t \geq 1, W^{s, t}(\Omega)$ signifies the standard Sobolev space of all functions whose (weak) derivatives up to order $s$ are bounded in the $L^{t}(\Omega)$-norm. Moreover, $W_{0}^{s, t}(\Omega)$ is the subspace of functions belonging to $W^{s, t}(\Omega)$ with zero trace along the boundary $\partial \Omega$. If $t=2$, we simply write $H^{s}(\Omega)=W^{s, 2}(\Omega)$. Following [3], Section 2, the above weak formulation is well-posed.

Second-order elliptic partial differential equations of the form (1.1)-(1.2) are employed, for instance, in the modelling of diffusion processes, heat flow, structural mechanics applications, or electric potentials, whenever point sources or loads occur. In addition, problems with a $\delta$-source appear as dual problems in deriving pointwise error estimates for finite element discretizations; see, e.g., $[6,10,12]$. From an analytical point of view, the challenge in describing such problems in a proper manner lies in the fact that the Dirac $\delta$-distribution in $\mathbb{R}^{2}$ does not belong to $H^{-1}(\Omega)$; thereby, the solution of (1.1)-(1.2) is not an $H^{1}$-function. Consequently, the numerical approximation of (1.1)-(1.2) by, for example, finite element methods, requires a non-standard analysis. Here, in the context of conforming FEM, we mention the a priori results in $[7,17]$, as well as the a posteriori error analysis in [3]. For DG approximations to low-regularity problems, see, e.g., [13,19].

The focus of the current paper is to extend some of the results developed for standard FEM to the context of discontinuous Galerkin methods. In particular, we shall derive a priori, as well as residual-based (global upper and local lower) a posteriori error estimates with respect to the $L^{2}$-norm. Whilst striving to keep matters rather general, we will use the symmetric interior penalty discontinuous Galerkin method (SIPG), see [4,9, 18], as an example to illustrate our results. To the authors knowledge, the analytical results contained in this article represent the first attempt to analyze DG methods for the numerical approximation of second-order elliptic partial differential equations with a Dirac delta source.

The outline of the article is as follows: in Section 2, we recall some basic definitions for discontinuous Galerkin discretizations. Then, in Section 3 the a priori error analysis of a general class of DG methods on quasi-uniform meshes is presented. Section 4 presents the residual-based a posteriori error analysis. Subsequently, in Section 5 numerical experiments are undertaken to confirm the theoretical results. Finally, in Section 6 we add some concluding remarks.

\section{Discontinuous Galerkin methods}

In this paper, we are interested in solving (1.1)-(1.2) numerically by means of suitable discontinuous Galerkin discretizations. Before discussing these schemes, we will first introduce a suitable finite element mesh framework for them.

\subsection{Meshes, spaces, and element boundary operators}

We consider shape-regular meshes $\mathcal{T}$ that partition $\Omega$ into open affine disjoint triangular or quadrilateral elements $\{K\}_{K \in \mathcal{T}}$, i.e., $\bar{\Omega}=\bigcup_{K \in \mathcal{T}} \bar{K}$. We suppose that $\mathcal{T}$ is constructed in such a manner that $\boldsymbol{x}_{0}$ lies in the interior of some element $K_{0} \in \mathcal{T}$. Furthermore, we permit meshes to be 1-irregular. Each element $K \in \mathcal{T}$ is an image of the open reference triangle $\widehat{T}=\left\{\left(\widehat{x}_{1}, \widehat{x}_{2}\right):-1<\widehat{x}_{1}<1,-1<\widehat{x}_{2}<-\widehat{x}_{1}\right\}$ or of the open reference square $\widehat{Q}=(-1,1)^{2}$, respectively. By $h_{K}$, we denote the diameter of an element $K \in \mathcal{T}$; the elemental diameters are stored in a vector $\boldsymbol{h}=\left[h_{K}\right]_{K \in \mathcal{T}}$.

Moreover, we will define some suitable element boundary operators that are required for DG methods. To this end, we denote by $\mathcal{E}_{\mathcal{I}}$ the set of all interior edges and by $\mathcal{E}_{\mathcal{B}}$ the set of all boundary edges in $\mathcal{T}$. Additionally, we set $\mathcal{E}=\mathcal{E}_{\mathcal{I}} \cup \mathcal{E}_{\mathcal{B}}$. The boundary $\partial K$ of an element $K$ and the sets $\partial K \backslash \partial \Omega$ and $\partial K \cap \partial \Omega$ will be identified in a natural way with the corresponding subsets of $\mathcal{E}$.

Let $K_{\sharp}$ and $K_{b}$ be two adjacent elements of $\mathcal{T}$, and $\boldsymbol{x}$ an arbitrary point on the interior edge $e \in \mathcal{E}_{\mathcal{I}}$ given by $e=\partial K_{\sharp} \cap \partial K_{\mathrm{b}}$. Furthermore, let $v$ and $\boldsymbol{q}$ be scalar- and vector-valued functions, respectively, that are sufficiently smooth inside each element $K_{\sharp / b}$. By $\left(v_{\sharp / b}, \boldsymbol{q}_{\sharp / b}\right)$, we denote the traces of $(v, \boldsymbol{q})$ on $e$ taken from within the interior of $K_{\sharp / b}$, respectively. Then, the averages of $v$ and $\boldsymbol{q}$ at $\boldsymbol{x} \in e$ are given by

$$
\langle\langle v\rangle\rangle=\frac{1}{2}\left(v_{\sharp}+v_{b}\right), \quad\langle\langle\boldsymbol{q}\rangle\rangle=\frac{1}{2}\left(\boldsymbol{q}_{\sharp}+\boldsymbol{q}_{b}\right),
$$


respectively. Similarly, the jumps of $v$ and $\boldsymbol{q}$ at $\boldsymbol{x} \in e$ are given by

$$
\llbracket v \rrbracket=v_{\sharp} \boldsymbol{n}_{K_{\sharp}}+v_{b} \boldsymbol{n}_{K_{b}}, \quad \llbracket \boldsymbol{q} \rrbracket=\boldsymbol{q}_{\sharp} \cdot \boldsymbol{n}_{K_{\sharp}}+\boldsymbol{q}_{b} \cdot \boldsymbol{n}_{K_{b}},
$$

respectively. Here, for $K \in \mathcal{T}$, we denote by $\boldsymbol{n}_{K}$ the unit outward normal vector to $\partial K$. On a boundary edge $e \in \mathcal{E}_{\mathcal{B}}$, we set $\langle\langle v\rangle\rangle=v,\langle\langle\boldsymbol{q}\rangle\rangle=\boldsymbol{q}$ and $\llbracket v \rrbracket=v \boldsymbol{n}$, with $\boldsymbol{n}$ denoting the unit outward normal vector on the boundary $\partial \Omega$.

\subsection{DG discretizations}

For a given finite element mesh $\mathcal{T}$ and a fixed polynomial degree $\ell \geq 1$, let us consider the DG finite element space

$$
V_{\mathrm{DG}}(\mathcal{T})=\left\{v \in L^{2}(\Omega):\left.v\right|_{K} \in \mathbb{S}_{\ell}(K) \quad \forall K \in \mathcal{T}\right\},
$$

where, for $K \in \mathcal{T}, \mathbb{S}_{\ell}(K)$ signifies either the space $\mathbb{P}_{\ell}(K)$ of all polynomials of total degree at most $\ell$ on $K$, when $K$ is a triangle, or the space $\mathbb{Q}_{\ell}(K)$ of all polynomials of degree at most $\ell$ in each coordinate direction, when $K$ is a quadrilateral.

Let us now consider a DG bilinear form $a_{\mathrm{DG}}(\cdot, \cdot)$ which discretizes the problem (1.1)-(1.2), i.e., we seek a DG solution $u_{\mathrm{DG}} \in V_{\mathrm{DG}}(\mathcal{T})$ such that

$$
a_{\mathrm{DG}}\left(u_{\mathrm{DG}}, v\right)=v\left(\boldsymbol{x}_{0}\right) \quad \forall v \in V_{\mathrm{DG}}(\mathcal{T}) .
$$

We assume that the matrix corresponding to $a_{\mathrm{DG}}(\cdot, \cdot)$ on $V_{\mathrm{DG}}(\mathcal{T}) \times V_{\mathrm{DG}}(\mathcal{T})$ is non-singular, so that the discrete solution $u_{\mathrm{DG}}$ is uniquely defined. Moreover, we suppose that $a_{\mathrm{DG}}(\cdot, \cdot)$ is of the form

$$
a_{\mathrm{DG}}(w, v)=\int_{\Omega} \nabla_{h} w \cdot \nabla_{h} v \mathrm{~d} \boldsymbol{x}+\mathcal{F}(w, v),
$$

where $\nabla_{h}$ denotes the elementwise gradient, and $\mathcal{F}(\cdot, \cdot)$ is a bilinear form featuring the numerical fluxes of the DG scheme under consideration.

In order to give an example, we recall the symmetric interior penalty discontinuous Galerkin method (SIPG); see, e.g., $[4,5,15,18]$. More precisely, for a fixed parameter $\gamma>0$, we define the DG form

$$
a_{\mathrm{DG}}(w, v)=\int_{\Omega} \nabla_{h} w \cdot \nabla_{h} v \mathrm{~d} \boldsymbol{x}-\int_{\mathcal{E}}\left\langle\left\langle\nabla_{h} w\right\rangle\right\rangle \cdot \llbracket v \rrbracket \mathrm{d} s-\int_{\mathcal{E}} \llbracket w \rrbracket \cdot\left\langle\left\langle\nabla_{h} v\right\rangle\right\rangle \mathrm{d} s+\gamma \int_{\mathcal{E}} \mathrm{h}^{-1} \llbracket w \rrbracket \cdot \llbracket v \rrbracket \mathrm{d} s .
$$

Here, $\mathrm{h} \in L^{\infty}(\mathcal{E})$ is given by

$$
\mathrm{h}(\boldsymbol{x})= \begin{cases}\min \left(h_{K_{\sharp}}, h_{K_{\mathrm{b}}}\right) & \text { for } \boldsymbol{x} \in \partial K_{\sharp} \cap \partial K_{b} \in \mathcal{E}_{\mathcal{I}}, \\ h_{K} & \text { for } \boldsymbol{x} \in \partial K \cap \partial \Omega \in \mathcal{E}_{\mathcal{B}} .\end{cases}
$$

For sufficiently large $\gamma>0$, the form $a_{\mathrm{DG}}(\cdot, \cdot)$ is coercive with respect to a suitable DG energy norm and hence, using the SIPG form (2.4) in (2.2), the matrix corresponding to the bilinear form $a_{\mathrm{DG}}(\cdot, \cdot)$ is invertible; $c f$., e.g., $[16]$.

\section{Convergence Behavior ON QUASI-Uniform MESHES}

The aim of this section is to prove an a priori error estimate for the DG method (2.2) with respect to the $L^{2}$-norm. To this end, let us suppose that the mesh $\mathcal{T}$ is quasi-uniform, with mesh size $h:=\max _{K \in \mathcal{T}} h_{K}$, that is, there exists a constant $\rho \geq 1$ such that $h \leq \rho h_{K}$, for any element $K \in \mathcal{T}$. 


\subsection{A discrete $\delta$-function}

Following the approach [17], we commence by constructing a discrete approximation $\delta_{h} \in V_{\mathrm{DG}}(\mathcal{T})$ of the Dirac delta function $\delta_{\boldsymbol{x}_{0}}$. More precisely, let

$$
\delta_{h}:= \begin{cases}0 & \text { on } \Omega \backslash \bar{K}_{0}, \\ \delta_{K_{0}} & \text { on } K_{0},\end{cases}
$$

where $K_{0} \in \mathcal{T}$ is the unique element which $\boldsymbol{x}_{0}$ belongs to. We define $\delta_{K_{0}} \in \mathbb{S}_{\ell}\left(K_{0}\right)$ by

$$
\int_{K_{0}} \delta_{K_{0}} v \mathrm{~d} \boldsymbol{x}=v\left(\boldsymbol{x}_{0}\right) \quad \forall v \in \mathbb{S}_{\ell}\left(K_{0}\right)
$$

Clearly, we have that

$$
\int_{\Omega} \delta_{h} v \mathrm{~d} \boldsymbol{x}=v\left(\boldsymbol{x}_{0}\right)
$$

for any $v \in V_{\mathrm{DG}}(\mathcal{T})$. We now write $\Pi_{K_{0}}^{\ell}$ to be the $L^{2}$-projection operator onto $\mathbb{S}_{\ell}\left(K_{0}\right)$; more precisely, given $w \in L^{2}\left(K_{0}\right)$, we define $\Pi_{K_{0}}^{\ell} w \in \mathbb{S}_{\ell}\left(K_{0}\right)$ as follows:

$$
\int_{K_{0}}\left(w-\Pi_{K_{0}}^{\ell} w\right) v \mathrm{~d} \boldsymbol{x}=0 \quad \forall v \in \mathbb{S}_{\ell}\left(K_{0}\right) .
$$

Thereby,

$$
\left\|\delta_{h}\right\|_{L^{2}(\Omega)}=\sup _{\substack{v \in L^{2}\left(K_{0}\right) \\ v \neq 0}} \frac{\int_{K_{0}} \delta_{h} v \mathrm{~d} \boldsymbol{x}}{\|v\|_{L^{2}\left(K_{0}\right)}}=\sup _{\substack{v \in L^{2}\left(K_{0}\right) \\ \neq \neq 0}} \frac{\int_{K_{0}} \delta_{h} \Pi_{K_{0}}^{\ell} v \mathrm{~d} \boldsymbol{x}}{\|v\|_{L^{2}\left(K_{0}\right)}} .
$$

Now, using that $\|w\|_{L^{2}\left(K_{0}\right)} \geq\left\|\Pi_{K_{0}}^{\ell} w\right\|_{L^{2}\left(K_{0}\right)}$ for any $w \in L^{2}\left(K_{0}\right)$, we obtain

$$
\left\|\delta_{h}\right\|_{L^{2}(\Omega)} \leq \sup _{\substack{v \in L^{2}\left(K_{0}\right) \\ \Pi_{K_{0}}^{\ell} v \neq 0}} \frac{\int_{K_{0}} \delta_{h} \Pi_{K_{0}}^{\ell} v \mathrm{~d} \boldsymbol{x}}{\left\|\Pi_{K_{0}}^{\ell} v\right\|_{L^{2}\left(K_{0}\right)}}=\sup _{\substack{v \in L^{2}\left(K_{0}\right) \\ \Pi_{K_{0}}^{\ell} v \neq 0}} \frac{\left|\Pi_{K_{0}}^{\ell} v\left(\boldsymbol{x}_{0}\right)\right|}{\left\|\Pi_{K_{0}}^{\ell} v\right\|_{L^{2}\left(K_{0}\right)}} \leq \sup _{\substack{v \in L^{2}\left(K_{0}\right) \\ \Pi_{K_{0}}^{\ell} v \neq 0}} \frac{\left\|\Pi_{K_{0}}^{\ell} v\right\|_{L^{\infty}\left(K_{0}\right)}}{\left\|\Pi_{K_{0}}^{\ell} v\right\|_{L^{2}\left(K_{0}\right)}}
$$

Furthermore, employing the inverse estimate

$$
\|w\|_{L^{\infty}\left(K_{0}\right)} \leq C h_{K_{0}}^{-1}\|w\|_{L^{2}\left(K_{0}\right)} \quad \forall w \in \mathbb{S}_{\ell}\left(K_{0}\right),
$$

it follows that

$$
\left\|\delta_{h}\right\|_{L^{2}(\Omega)} \leq C h_{K_{0}}^{-1} .
$$

In addition, letting $v \equiv 1$ in (3.3) leads to

$$
\left\|\delta_{h}\right\|_{L^{2}(\Omega)} \geq \frac{v\left(\boldsymbol{x}_{0}\right)}{\|v\|_{L^{2}\left(K_{0}\right)}}=\frac{1}{\|1\|_{L^{2}\left(K_{0}\right)}} \geq C h_{K_{0}}^{-1} .
$$

\subsection{A priori error analysis}

The function $\delta_{h}$ from (3.1) is used to define the ensuing auxiliary problem:

$$
\begin{aligned}
-\Delta U^{h} & =\delta_{h} & & \text { in } \Omega, \\
U^{h} & =0 & & \text { on } \partial \Omega .
\end{aligned}
$$


The standard weak formulation is to find $U^{h} \in H_{0}^{1}(\Omega)$ such that

$$
a\left(U^{h}, v\right)=\int_{\Omega} \delta_{h} v \mathrm{~d} \boldsymbol{x} \quad \forall v \in H_{0}^{1}(\Omega) .
$$

Since $\Omega$ is convex, the Laplace operator $\Delta: H^{2}(\Omega) \cap H_{0}^{1}(\Omega) \rightarrow L^{2}(\Omega)$ is an isomorphism; see, e.g., [8, 11]. In particular,

$$
\left\|\Delta^{-1}\right\|_{L^{2}(\Omega) \rightarrow H^{2}(\Omega) \cap H_{0}^{1}(\Omega)}<\infty .
$$

Thus, we have

$$
\left\|U^{h}\right\|_{H^{2}(\Omega)} \leq C\left\|\delta_{h}\right\|_{L^{2}(\Omega)} .
$$

Referring to [17], the following error bound holds

$$
\left\|u-U^{h}\right\|_{L^{2}(\Omega)} \leq C h,
$$

where $u$ is the solution of (1.1)-(1.2), and $C>0$ is a constant depending on the distance of $\boldsymbol{x}_{0}$ to $\partial \Omega$.

In addition, using (3.1), we notice that the DG solution $u_{\mathrm{DG}}$ from (2.2) satisfies

$$
a_{\mathrm{DG}}\left(u_{\mathrm{DG}}, v\right)=v\left(\boldsymbol{x}_{0}\right)=\int_{\Omega} \delta_{h} v \mathrm{~d} \boldsymbol{x}
$$

for any $v \in V_{\mathrm{DG}}(\mathcal{T})$. Consequently, $u_{\mathrm{DG}}$ can be seen to be the DG approximation of $U^{h}$. Hence, provided that (3.7) holds, we may assume that we have the estimate

$$
\left\|U^{h}-u_{\mathrm{DG}}\right\|_{L^{2}(\Omega)} \leq C h^{2}\left\|U^{h}\right\|_{H^{2}(\Omega)} .
$$

Indeed, this bound is true for various DG schemes in the literature (such as, for instance, the SIPG method (2.4)); see [5]. Thus, employing (3.5) we conclude that

$$
\left\|U^{h}-u_{\mathrm{DG}}\right\|_{L^{2}(\Omega)} \leq C h^{2}\left\|\delta_{h}\right\|_{L^{2}(\Omega)} \leq C h .
$$

Thereby, exploiting the triangle inequality, gives

$$
\left\|u-u_{\mathrm{DG}}\right\|_{L^{2}(\Omega)} \leq\left\|u-U^{h}\right\|_{L^{2}(\Omega)}+\left\|U^{h}-u_{\mathrm{DG}}\right\|_{L^{2}(\Omega)} ;
$$

inserting the bounds (3.9) and (3.11) into (3.12), we deduce the following result.

Theorem 3.1. Let $\mathcal{T}$ be a quasi-uniform mesh of mesh size $h$. Furthermore, suppose that (3.7), as well as the $L^{2}$-error estimate (3.10) hold. Then, we have the following a priori error bound

$$
\left\|u-u_{\mathrm{DG}}\right\|_{L^{2}(\Omega)} \leq C h,
$$

where $u$ and $u_{\mathrm{DG}}$ are the solutions of (1.1)-(1.2) and (2.2), respectively, and $C>0$ is a constant independent of $h$.

Remark 3.2. We remark that the above error bound may be improved on meshes that are appropriately graded about the point $\boldsymbol{x}_{0}$; see [2].

\section{ResiduAl-BASEd A POSTERIORI ERROR ANALYSiS}

We now proceed by developing an $L^{2}$-norm a posteriori error analysis of the DG schemes defined in (2.2). Here, we derive both general upper and (local) lower bounds on the error measured in terms of the $L^{2}$-norm. Additionally, in order to present a specific example, the general results will be applied to the SIPG method. 


\subsection{Upper bound}

For any $p \in L^{2}(\Omega)$, let us consider the dual problem

$$
\begin{aligned}
-\Delta \psi=p & \text { in } \Omega, \\
\psi=0 & \text { on } \partial \Omega .
\end{aligned}
$$

The weak formulation reads: find $\psi \in H_{0}^{1}(\Omega)$ such that

$$
a(\psi, v)=\int_{\Omega} p v \mathrm{~d} \boldsymbol{x} \quad \forall v \in H_{0}^{1}(\Omega),
$$

where $a(\cdot, \cdot)$ is the bilinear form defined in (1.3). By (3.7), we have the elliptic regularity estimate

$$
\|\psi\|_{H^{2}(\Omega)} \leq C\|p\|_{L^{2}(\Omega)} .
$$

For the $L^{2}$-norm of the error $u-u_{\mathrm{DG}}$ in the DG discretization, we may write

$$
\left\|u-u_{\mathrm{DG}}\right\|_{L^{2}(\Omega)}=\sup _{\substack{p \in L^{2}(\Omega) \\ p \neq 0}} \frac{\int_{\Omega}\left(u-u_{\mathrm{DG}}\right) p \mathrm{~d} \boldsymbol{x}}{\|p\|_{L^{2}(\Omega)}} .
$$

Here, for the integral we have

$$
\int_{\Omega}\left(u-u_{\mathrm{DG}}\right) p \mathrm{~d} \boldsymbol{x}=a(u, \psi)+\int_{\Omega} u_{\mathrm{DG}} \Delta \psi \mathrm{d} \boldsymbol{x}=\psi\left(\boldsymbol{x}_{0}\right)+\int_{\Omega} u_{\mathrm{DG}} \Delta \psi \mathrm{d} \boldsymbol{x} .
$$

Twofold integration by parts (element by element) of the last term results in

$$
\begin{aligned}
\int_{\Omega} u_{\mathrm{DG}} \Delta \psi \mathrm{d} \boldsymbol{x} & =-\sum_{K \in \mathcal{T}} \int_{K} \nabla u_{\mathrm{DG}} \cdot \nabla \psi \mathrm{d} \boldsymbol{x}+\sum_{K \in \mathcal{T}} \int_{\partial K}\left(\nabla \psi \cdot \boldsymbol{n}_{K}\right) u_{\mathrm{DG}} \mathrm{d} s \\
& =\sum_{K \in \mathcal{T}} \int_{K} \psi \Delta u_{\mathrm{DG}} \mathrm{d} \boldsymbol{x}-\sum_{K \in \mathcal{T}} \int_{\partial K}\left(\nabla u_{\mathrm{DG}} \cdot \boldsymbol{n}_{K}\right) \psi \mathrm{d} s+\sum_{K \in \mathcal{T}} \int_{\partial K}\left(\nabla \psi \cdot \boldsymbol{n}_{K}\right) u_{\mathrm{DG}} \mathrm{d} s .
\end{aligned}
$$

Furthermore, applying some elementary calculations, we obtain

$$
\int_{\Omega} u_{\mathrm{DG}} \Delta \psi \mathrm{d} \boldsymbol{x}=\sum_{K \in \mathcal{T}} \int_{K} \psi \Delta u_{\mathrm{DG}} \mathrm{d} \boldsymbol{x}-\int_{\mathcal{E}_{\mathcal{I}}} \llbracket \nabla_{h} u_{\mathrm{DG}} \rrbracket \psi \mathrm{d} s+\int_{\mathcal{E}} \llbracket u_{\mathrm{DG}} \rrbracket \cdot \nabla \psi \mathrm{d} s .
$$

For any $\psi_{h} \in V_{\mathrm{DG}}(\mathcal{T})$, there holds

$$
\psi_{h}\left(\boldsymbol{x}_{0}\right)=a_{\mathrm{DG}}\left(u_{\mathrm{DG}}, \psi_{h}\right)=\int_{\Omega} \nabla_{h} u_{\mathrm{DG}} \cdot \nabla_{h} \psi_{h} \mathrm{~d} \boldsymbol{x}+\mathcal{F}\left(u_{\mathrm{DG}}, \psi_{h}\right) ;
$$

$c f$. (2.3). An elementwise integration by parts and elementary manipulations as before, yield that

$$
\begin{aligned}
\int_{\Omega} \nabla_{h} u_{\mathrm{DG}} \cdot \nabla_{h} \psi_{h} \mathrm{~d} \boldsymbol{x} & =-\sum_{K \in \mathcal{T}} \int_{K} \psi_{h} \Delta u_{\mathrm{DG}} \mathrm{d} \boldsymbol{x}+\sum_{K \in \mathcal{T}} \int_{\partial K}\left(\nabla u_{\mathrm{DG}} \cdot \boldsymbol{n}_{K}\right) \psi_{h} \mathrm{~d} s \\
& =-\sum_{K \in \mathcal{T}} \int_{K} \psi_{h} \Delta u_{\mathrm{DG}} \mathrm{d} \boldsymbol{x}+\int_{\mathcal{E}}\left\langle\left\langle\nabla_{h} u_{\mathrm{DG}}\right\rangle\right\rangle \cdot \llbracket \psi_{h} \rrbracket \mathrm{d} s+\int_{\mathcal{E}_{\mathcal{I}}} \llbracket \nabla_{h} u_{\mathrm{DG}} \rrbracket\left\langle\left\langle\psi_{h}\right\rangle\right\rangle \mathrm{d} s .
\end{aligned}
$$


Therefore, we obtain that

$$
\begin{aligned}
\int_{\Omega}\left(u-u_{\mathrm{DG}}\right) p \mathrm{~d} \boldsymbol{x}= & \left(\psi-\psi_{h}\right)\left(\boldsymbol{x}_{0}\right)+\sum_{K \in \mathcal{T}} \int_{K}\left(\psi-\psi_{h}\right) \Delta u_{\mathrm{DG}} \mathrm{d} \boldsymbol{x} \\
& \left.-\int_{\mathcal{E}_{\mathcal{I}}} \llbracket \nabla_{h} u_{\mathrm{DG}} \rrbracket\left\langle\psi-\psi_{h}\right\rangle\right\rangle \mathrm{d} s+\mathcal{R}\left[u_{\mathrm{DG}}, \psi\right]\left(\psi_{h}\right),
\end{aligned}
$$

where

$$
\mathcal{R}\left[u_{\mathrm{DG}}, \psi\right]\left(\psi_{h}\right)=\int_{\mathcal{E}}\left\langle\left\langle\nabla_{h} u_{\mathrm{DG}}\right\rangle\right\rangle \cdot \llbracket \psi_{h} \rrbracket \mathrm{d} s+\int_{\mathcal{E}} \llbracket u_{\mathrm{DG}} \rrbracket \cdot \nabla \psi \mathrm{d} s+\mathcal{F}\left(u_{\mathrm{DG}}, \psi_{h}\right)
$$

is a residual term. We make the assumption that

$$
\left|\mathcal{R}\left[u_{\mathrm{DG}}, \psi\right]\left(\psi_{h}\right)\right| \leq C \Upsilon\left(u_{\mathrm{DG}}\right)\left\|\psi-\psi_{h}\right\|_{h},
$$

where $C>0$ is a constant independent of $\boldsymbol{h}, \Upsilon\left(u_{\mathrm{DG}}\right)$ is a computable quantity, and $\|\cdot\|_{h}$ is a semi-norm such that we can find an interpolant $\psi_{h} \in V_{\mathrm{DG}}(\mathcal{T})$ of the solution $\psi$ of $(4.1)-(4.2)$ with

$$
h_{K_{0}}^{-2} \sup _{\boldsymbol{x} \in K_{0}}\left|\left(\psi-\psi_{h}\right)(\boldsymbol{x})\right|^{2}+\sum_{K \in \mathcal{T}} h_{K}^{-4}\left\|\psi-\psi_{h}\right\|_{L^{2}(K)}^{2}+\sum_{K \in \mathcal{T}} h_{K}^{-2}\left\|\nabla\left(\psi-\psi_{h}\right)\right\|_{L^{2}(K)}^{2}+\left\|\psi-\psi_{h}\right\|_{h}^{2} \leq C\|\psi\|_{H^{2}(\Omega)}^{2},
$$

for a constant $C>0$ independent of $\boldsymbol{h}$. Here, $K_{0} \in \mathcal{T}$ is again the element containing the point $\boldsymbol{x}_{0}$ which the $\delta$-distribution $\delta_{\boldsymbol{x}_{0}}$ from (1.1) is centered at.

In order to proceed, we recall the $L^{2}$-projection onto $\mathbb{S}_{\ell}\left(K_{0}\right)$ from (3.2). Then, applying (3.1), gives

$$
\begin{aligned}
\left(\psi-\psi_{h}\right)\left(\boldsymbol{x}_{0}\right) & =\left(\psi-\Pi_{K_{0}}^{\ell} \psi\right)\left(\boldsymbol{x}_{0}\right)+\Pi_{K_{0}}^{\ell}\left(\psi-\psi_{h}\right)\left(\boldsymbol{x}_{0}\right) \\
& =\left(\psi-\Pi_{K_{0}}^{\ell} \psi\right)\left(\boldsymbol{x}_{0}\right)+\int_{K_{0}} \Pi_{K_{0}}^{\ell}\left(\psi-\psi_{h}\right) \delta_{h} \mathrm{~d} \boldsymbol{x} \\
& =\left(\psi-\Pi_{K_{0}}^{\ell} \psi\right)\left(\boldsymbol{x}_{0}\right)+\int_{K_{0}}\left(\psi-\psi_{h}\right) \delta_{h} \mathrm{~d} \boldsymbol{x} .
\end{aligned}
$$

Hence,

$$
\int_{\Omega}\left(u-u_{\mathrm{DG}}\right) p \mathrm{~d} \boldsymbol{x}=\left(\psi-\Pi_{K_{0}}^{\ell} \psi\right)\left(\boldsymbol{x}_{0}\right)+\sum_{K \in \mathcal{T}} \int_{K}\left(\psi-\psi_{h}\right)\left(\Delta u_{\mathrm{DG}}+\delta_{h}\right) \mathrm{d} \boldsymbol{x}-\int_{\mathcal{E}_{\mathcal{I}}} \llbracket \nabla_{h} u_{\mathrm{DG}} \rrbracket\left\langle\left\langle\psi-\psi_{h}\right\rangle\right\rangle \mathrm{d} s+\mathcal{R}\left[u_{\mathrm{DG}}, \psi\right]\left(\psi_{h}\right) .
$$

Therefore, using (4.6), it follows that

$$
\begin{aligned}
& \left|\int_{\Omega}\left(u-u_{\mathrm{DG}}\right) p \mathrm{~d} \boldsymbol{x}\right| \leq \sup _{\boldsymbol{x} \in K_{0}}\left|\left(\Pi_{K_{0}}^{\ell} \psi-\psi\right)(\boldsymbol{x})\right|+\sum_{K \in \mathcal{T}}\left\|\psi-\psi_{h}\right\|_{L^{2}(K)}\left\|\Delta u_{\mathrm{DG}}+\delta_{h}\right\|_{L^{2}(K)} \\
& +\left\|\mathrm{h}^{\frac{3}{2}} \llbracket \nabla_{h} u_{\mathrm{DG}} \rrbracket\right\|_{L^{2}\left(\mathcal{E}_{\mathcal{I}}\right)}\left\|\mathrm{h}^{-\frac{3}{2}}\left\langle\left\langle\psi-\psi_{h}\right\rangle\right\rangle\right\|_{L^{2}\left(\mathcal{E}_{\mathcal{I}}\right)}+C \Upsilon\left(u_{\mathrm{DG}}\right)\left\|\psi-\psi_{h}\right\|_{h} \\
& \leq C\left(h_{K_{0}}^{2}+\sum_{K \in \mathcal{T}} h_{K}^{4}\left\|\Delta u_{\mathrm{DG}}+\delta_{h}\right\|_{L^{2}(K)}^{2}+\left\|\mathrm{h}^{\frac{3}{2}} \llbracket \nabla_{h} u_{\mathrm{DG}} \rrbracket\right\|_{L^{2}\left(\mathcal{E}_{\mathcal{I}}\right)}^{2}+\Upsilon\left(u_{\mathrm{DG}}\right)^{2}\right)^{\frac{1}{2}} \\
& \times\left(h_{K_{0}}^{-2} \sup _{\boldsymbol{x} \in K_{0}}\left|\left(\psi-\Pi_{K_{0}}^{\ell} \psi\right)(\boldsymbol{x})\right|^{2}+\sum_{K \in \mathcal{T}} h_{K}^{-4}\left\|\psi-\psi_{h}\right\|_{L^{2}(K)}^{2}+\| \mathrm{h}^{-\frac{3}{2}}\left\langle\left\langle\psi-\psi_{h}\right\rangle\left\|_{L^{2}\left(\mathcal{E}_{\mathcal{I}}\right)}^{2}+\right\| \psi-\psi_{h} \|_{h}^{2}\right)^{\frac{1}{2}} .\right.
\end{aligned}
$$

Here, employing a standard trace inequality, we notice that

$$
\begin{aligned}
\| \mathrm{h}^{-\frac{3}{2}}\left\langle\left\langle\psi-\psi_{h}\right\rangle \|_{L^{2}\left(\mathcal{E}_{\mathcal{I}}\right)}^{2}\right. & \leq C \sum_{K \in \mathcal{T}} h_{K}^{-3}\left\|\psi-\psi_{h}\right\|_{L^{2}(\partial K \backslash \partial \Omega)}^{2} \\
& \leq C \sum_{K \in \mathcal{T}}\left(h_{K}^{-4}\left\|\psi-\psi_{h}\right\|_{L^{2}(K)}^{2}+h_{K}^{-2}\left\|\nabla\left(\psi-\psi_{h}\right)\right\|_{L^{2}(K)}^{2}\right) .
\end{aligned}
$$


Furthermore,

$$
\sup _{\boldsymbol{x} \in K_{0}}\left|\left(\psi-\Pi_{K_{0}}^{\ell} \psi\right)(\boldsymbol{x})\right| \leq \sup _{\boldsymbol{x} \in K_{0}}\left|\left(\psi-\psi_{h}\right)(\boldsymbol{x})\right|+\sup _{\boldsymbol{x} \in K_{0}}\left|\Pi_{K_{0}}^{\ell}\left(\psi-\psi_{h}\right)(\boldsymbol{x})\right| .
$$

Applying the inverse estimate (3.4), leads to

$$
\sup _{\boldsymbol{x} \in K_{0}}\left|\Pi_{K_{0}}^{\ell}\left(\psi-\psi_{h}\right)(\boldsymbol{x})\right| \leq C h_{K_{0}}^{-1}\left\|\Pi_{K_{0}}^{\ell}\left(\psi-\psi_{h}\right)\right\|_{L^{2}\left(K_{0}\right)} \leq C h_{K_{0}}^{-1}\left\|\psi-\psi_{h}\right\|_{L^{2}\left(K_{0}\right)} .
$$

It follows that

$$
\begin{aligned}
& \left|\int_{\Omega}\left(u-u_{\mathrm{DG}}\right) p \mathrm{~d} \boldsymbol{x}\right| \leq C\left(h_{K_{0}}^{2}+\sum_{K \in \mathcal{T}} h_{K}^{4}\left\|\Delta u_{\mathrm{DG}}+\delta_{h}\right\|_{L^{2}(K)}^{2}+\sum_{K \in \mathcal{T}} h_{K}^{3}\left\|\llbracket \nabla_{h} u_{\mathrm{DG}} \rrbracket\right\|_{L^{2}(\partial K \backslash \partial \Omega)}^{2}+\Upsilon\left(u_{\mathrm{DG}}\right)^{2}\right)^{\frac{1}{2}} \\
& \quad \times\left(h_{K_{0}}^{-2} \sup _{\boldsymbol{x} \in K_{0}}\left|\left(\psi-\psi_{h}\right)(\boldsymbol{x})\right|^{2}+\sum_{K \in \mathcal{T}} h_{K}^{-4}\left\|\psi-\psi_{h}\right\|_{L^{2}(K)}^{2}+\sum_{K \in \mathcal{T}} h_{K}^{-2}\left\|\nabla\left(\psi-\psi_{h}\right)\right\|_{L^{2}(K)}^{2}+\left\|\psi-\psi_{h}\right\|_{h}^{2}\right)^{\frac{1}{2}} .
\end{aligned}
$$

Recalling (4.7), this becomes

$$
\left|\int_{\Omega}\left(u-u_{\mathrm{DG}}\right) p \mathrm{~d} \boldsymbol{x}\right| \leq C\left(h_{K_{0}}^{2}+\Upsilon\left(u_{\mathrm{DG}}\right)^{2}+\sum_{K \in \mathcal{T}} \widetilde{\eta}_{K}\right)^{\frac{1}{2}}\|\psi\|_{H^{2}(\Omega)},
$$

where, for each $K \in \mathcal{T}$, the local error indicator $\widetilde{\eta}_{K}$ is given by

$$
\widetilde{\eta}_{K}:=h_{K}^{4}\left\|\Delta u_{\mathrm{DG}}+\delta_{h}\right\|_{L^{2}(K)}^{2}+h_{K}^{3}\left\|\llbracket \nabla_{h} u_{\mathrm{DG}} \rrbracket\right\|_{L^{2}(\partial K \backslash \partial \Omega)}^{2} .
$$

Thereby, for any constant $\kappa>0$, defining the error indicators

$$
\eta_{\kappa, K}:=h_{K}^{4}\left\|\Delta u_{\mathrm{DG}}+\delta_{h}\right\|_{L^{2}(K)}^{2}+h_{K}^{3}\left\|\llbracket \nabla_{h} u_{\mathrm{DG}} \rrbracket\right\|_{L^{2}(\partial K \backslash \partial \Omega)}^{2}+\kappa^{2} h_{K}\left\|\llbracket u_{\mathrm{DG}} \rrbracket\right\|_{L^{2}(\partial K)}^{2},
$$

noting that $\left.\llbracket u \rrbracket\right|_{\mathcal{E}}=0$ (for $u \in W_{0}^{1, p}(\Omega)$ ), employing the elliptic regularity bound (4.3), and recalling (4.4), yields the following result.

Theorem 4.1. Let $u_{\mathrm{DG}}$ be the $D G$ solution given by (2.2) and $\psi$ be the solution of (4.1)-(4.2). Assume that the residual $\mathcal{R}\left[u_{\mathrm{DG}}, \psi\right]\left(\psi_{h}\right)$ defined in (4.5) satisfies (4.6) and (4.7) for some semi-norm $\|\cdot\|_{h}$ and some interpolant $\psi_{h} \in V_{\mathrm{DG}}(\mathcal{T})$. Then, the a posteriori error estimate holds

$$
\left\|u-u_{\mathrm{DG}}\right\|_{L^{2}(\Omega)}^{2}+\kappa^{2}\left\|\mathrm{~h}^{\frac{1}{2} \llbracket u-u_{\mathrm{DG}} \rrbracket}\right\|_{L^{2}(\mathcal{E})}^{2} \leq C\left(h_{K_{0}}^{2}+\Upsilon\left(u_{\mathrm{DG}}\right)^{2}+\sum_{K \in \mathcal{T}} \eta_{\kappa, K}\right),
$$

where $\eta_{\kappa, K}, K \in \mathcal{T}$, are the local error indicators defined in (4.8). The constant $C>0$ is independent of $\boldsymbol{h}$ and $\kappa$.

Remark 4.2. The two equivalent terms $\left\|\mathrm{h}^{\frac{1}{2}} \llbracket u-u_{\mathrm{DG}} \rrbracket\right\|_{L^{2}(\mathcal{E})}^{2}$ and $\sum_{K \in \mathcal{T}} h_{K}\left\|\llbracket u_{\mathrm{DG}} \rrbracket\right\|_{L^{2}(\partial K)}^{2}$ have been added on both sides of the a posteriori error estimate (4.9) since the extended $L^{2}$-norm

$$
\left\|u-u_{\mathrm{DG}}\right\|_{0, h}^{2} \equiv\left\|u-u_{\mathrm{DG}}\right\|_{L^{2}(\Omega)}^{2}+\kappa^{2}\left\|\mathrm{~h}^{\frac{1}{2}} \llbracket u-u_{\mathrm{DG}} \rrbracket\right\|_{L^{2}(\mathcal{E})}^{2}
$$

of the error appears to be a suitable norm for proving local lower a posteriori error estimates; see the subsequent section. 


\subsection{Local lower estimates}

Whilst our result in the previous section proves the reliability of the proposed a posteriori error estimator, we now focus on efficiency bounds in the sequel. We note that the convexity of the domain $\Omega$ is not required in this part of the article.

Let us consider the individual terms in the error indicator $\eta_{\kappa, K}, K \in \mathcal{T}$, from (4.8).

Proposition 4.3. For each $K \in \mathcal{T}$, the lower error bounds

$$
\left\|\Delta u_{\mathrm{DG}}+\delta_{h}\right\|_{L^{2}\left(K_{0}\right)} \leq C h_{K_{0}}^{-2}\left(\left\|\delta_{h}-\delta_{\boldsymbol{x}_{0}}\right\|_{H^{-2}(\Omega)}+\left\|u-u_{\mathrm{DG}}\right\|_{L^{2}\left(K_{0}\right)}\right),
$$

and

$$
\left\|\Delta u_{\mathrm{DG}}+\delta_{h}\right\|_{L^{2}(K)} \leq C h_{K}^{-2}\left\|u-u_{\mathrm{DG}}\right\|_{L^{2}(K)}, \quad K \in \mathcal{T} \backslash\left\{K_{0}\right\}
$$

hold.

Proof. For each element $K \in \mathcal{T}$ we define a smooth bubble function $b_{K}$ on $K$ that satisfies

$$
\operatorname{supp} b_{K} \subseteq K, \quad b_{K} \geq 0, \quad \sup _{\boldsymbol{x} \in K} b_{K}(\boldsymbol{x})=1,\left.\quad b_{K}\right|_{\partial K}=0,\left.\quad \nabla b_{K}\right|_{\partial K}=\mathbf{0} .
$$

Then, focusing on $K_{0}$ first and using the equivalence of norms in finite dimensional spaces, we have that

$$
C\left\|\Delta u_{\mathrm{DG}}+\delta_{h}\right\|_{L^{2}\left(K_{0}\right)}^{2} \leq \int_{K_{0}} v\left(\Delta u_{\mathrm{DG}}+\delta_{h}\right) \mathrm{d} \boldsymbol{x}=\int_{\Omega}\left(\delta_{h}-\delta_{\boldsymbol{x}_{0}}\right) v \mathrm{~d} \boldsymbol{x}+\int_{K_{0}} v \Delta\left(u_{\mathrm{DG}}-u\right) \mathrm{d} \boldsymbol{x},
$$

where $v:=b_{K}\left(\Delta u_{\mathrm{DG}}+\delta_{h}\right)$. Noticing that $\left.v\right|_{\partial K_{0}}=0$ and

$$
\left.\nabla v\right|_{\partial K_{0}}=\left.\left.b_{K}\right|_{\partial K_{0}} \nabla\left(\Delta u_{\mathrm{DG}}+\delta_{h}\right)\right|_{\partial K_{0}}+\left.\left.\nabla b_{K}\right|_{\partial K_{0}}\left(\Delta u_{\mathrm{DG}}+\delta_{h}\right)\right|_{\partial K_{0}}=\mathbf{0},
$$

integrating by parts twice in the second integral yields

$$
\begin{aligned}
C\left\|\Delta u_{\mathrm{DG}}+\delta_{h}\right\|_{L^{2}\left(K_{0}\right)}^{2} & \leq \int_{\Omega}\left(\delta_{h}-\delta_{\boldsymbol{x}_{0}}\right) v \mathrm{~d} \boldsymbol{x}+\int_{K_{0}} \Delta v\left(u_{\mathrm{DG}}-u\right) \mathrm{d} \boldsymbol{x} \\
& \leq\left(\left\|\delta_{h}-\delta_{\boldsymbol{x}_{0}}\right\|_{H^{-2}(\Omega)}+\left\|u-u_{\mathrm{DG}}\right\|_{L^{2}\left(K_{0}\right)}\right)\|v\|_{H^{2}\left(K_{0}\right)} .
\end{aligned}
$$

Again, due to equivalence of norms in finite dimensional spaces, and scaling, we have

$$
\|v\|_{H^{2}\left(K_{0}\right)} \leq C h_{K_{0}}^{-2}\|v\|_{L^{2}\left(K_{0}\right)} .
$$

Hence,

$$
\begin{aligned}
C\left\|\Delta u_{\mathrm{DG}}+\delta_{h}\right\|_{L^{2}\left(K_{0}\right)}^{2} & \leq h_{K_{0}}^{-2}\left(\left\|\delta_{h}-\delta_{\boldsymbol{x}_{0}}\right\|_{H^{-2}(\Omega)}+\left\|u-u_{\mathrm{DG}}\right\|_{L^{2}\left(K_{0}\right)}\right)\|v\|_{L^{2}\left(K_{0}\right)} \\
& \leq h_{K_{0}}^{-2}\left(\left\|\delta_{h}-\delta_{\boldsymbol{x}_{0}}\right\|_{H^{-2}(\Omega)}+\left\|u-u_{\mathrm{DG}}\right\|_{L^{2}\left(K_{0}\right)}\right)\left\|\Delta u_{\mathrm{DG}}+\delta_{h}\right\|_{L^{2}\left(K_{0}\right)} .
\end{aligned}
$$

Dividing both sides of the above inequality by $\left\|\Delta u_{\mathrm{DG}}+\delta_{h}\right\|_{L^{2}\left(K_{0}\right)}$ proves the proposition for $K_{0}$. 
For $K \in \mathcal{T} \backslash\left\{K_{0}\right\}$ we let $v=b_{K} \Delta u_{\mathrm{DG}}$ and notice that $\left.\delta_{h}\right|_{K}=0$ and $v\left(\boldsymbol{x}_{0}\right)=0$. Thence,

$$
C\left\|\Delta u_{\mathrm{DG}}+\delta_{h}\right\|_{L^{2}(K)}^{2}=C\left\|\Delta u_{\mathrm{DG}}\right\|_{L^{2}(K)}^{2} \leq \int_{K} v \Delta u_{\mathrm{DG}} \mathrm{d} \boldsymbol{x}=\int_{K} v \Delta\left(u_{\mathrm{DG}}-u\right) \mathrm{d} \boldsymbol{x} .
$$

The remainder of the proof is very similar as before.

Proposition 4.4. On $K_{0}$, the following local lower bound holds

$$
h_{K_{0}} \leq C\left(\left\|u-u_{\mathrm{DG}}\right\|_{L^{2}\left(K_{0}\right)}+\left\|\delta_{h}-\delta_{\boldsymbol{x}_{0}}\right\|_{H^{-2}(\Omega)}\right) .
$$

Proof. On the element $K_{0}$ consider a smooth bubble function $b_{K_{0}}$ that satisfies the properties (4.10) as well as

$$
b_{K_{0}}\left(\boldsymbol{x}_{0}\right)=1, \quad\left\|b_{K_{0}}\right\|_{L^{2}\left(K_{0}\right)} \leq \frac{1}{2}\left\|\delta_{h}\right\|_{L^{2}\left(K_{0}\right)}^{-1}=\mathcal{O}\left(h_{K_{0}}\right), \quad\left\|\Delta b_{K_{0}}\right\|_{L^{2}\left(K_{0}\right)} \leq C h_{K_{0}}^{-1} .
$$

Due to (3.5) and (3.6), this construction is possible by choosing a bubble function possessing a sufficiently small support in $K_{0}$. Then,

$$
1=\int_{\Omega} \delta_{\boldsymbol{x}_{0}} b_{K_{0}} \mathrm{~d} \boldsymbol{x}=\int_{K_{0}} \nabla\left(u-u_{\mathrm{DG}}\right) \cdot \nabla b_{K_{0}} \mathrm{~d} \boldsymbol{x}+\int_{K_{0}} \nabla u_{\mathrm{DG}} \cdot \nabla b_{K_{0}} \mathrm{~d} \boldsymbol{x} .
$$

Integration by parts, leads to

$$
\begin{aligned}
1 & =-\int_{K_{0}}\left(u-u_{\mathrm{DG}}\right) \Delta b_{K_{0}} \mathrm{~d} \boldsymbol{x}-\int_{K_{0}} b_{K_{0}} \Delta u_{\mathrm{DG}} \mathrm{d} \boldsymbol{x} \\
& =-\int_{K_{0}}\left(u-u_{\mathrm{DG}}\right) \Delta b_{K_{0}} \mathrm{~d} \boldsymbol{x}-\int_{K_{0}}\left(\delta_{h}+\Delta u_{\mathrm{DG}}\right) b_{K_{0}} \mathrm{~d} \boldsymbol{x}+\int_{K_{0}} \delta_{h} b_{K_{0}} \mathrm{~d} \boldsymbol{x} \\
& \leq\left\|u-u_{\mathrm{DG}}\right\|_{L^{2}\left(K_{0}\right)}\left\|\Delta b_{K_{0}}\right\|_{L^{2}\left(K_{0}\right)}+\left\|\delta_{h}+\Delta u_{\mathrm{DG}}\right\|_{L^{2}\left(K_{0}\right)}\left\|b_{K_{0}}\right\|_{L^{2}\left(K_{0}\right)}+\left\|\delta_{h}\right\|_{L^{2}\left(K_{0}\right)}\left\|b_{K_{0}}\right\|_{L^{2}\left(K_{0}\right)} \\
& \leq C h_{K_{0}}^{-1}\left\|u-u_{\mathrm{DG}}\right\|_{L^{2}\left(K_{0}\right)}+C h_{K_{0}}\left\|\delta_{h}+\Delta u_{\mathrm{DG}}\right\|_{L^{2}\left(K_{0}\right)}+\frac{1}{2} .
\end{aligned}
$$

This implies the bound

$$
h_{K_{0}} \leq C\left(\left\|u-u_{\mathrm{DG}}\right\|_{L^{2}\left(K_{0}\right)}+h_{K_{0}}^{2}\left\|\delta_{h}+\Delta u_{\mathrm{DG}}\right\|_{L^{2}\left(K_{0}\right)}\right) .
$$

Invoking the bound from Proposition 4.3 shows the estimate.

In order to bound the term $\left\|\llbracket \nabla_{h} u_{\mathrm{DG}} \rrbracket\right\|_{L^{2}(\partial K \backslash \partial \Omega)}$ from (4.8) we assume that the mesh $\mathcal{T}$ is regular (i.e., it does not contain any hanging nodes).

Proposition 4.5. Let $\mathcal{T}$ be regular. Consider two elements $K_{\sharp}, K_{b} \in \mathcal{T}$ that share an interface $e=\left(\partial K_{\sharp} \cap\right.$ $\left.\partial K_{b}\right)^{\circ} \in \mathcal{E}_{\mathcal{I}}$. We let $\omega_{e}:=\left(\bar{K}_{\sharp} \cup \bar{K}_{b}\right)^{\circ}$. Then, the lower bound holds

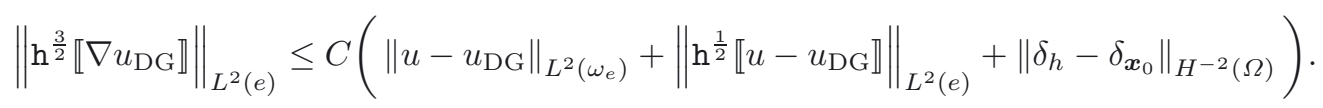

Proof. Pursuing a similar approach as in $[13,14]$ (i.e., by choosing a suitable smooth nonnegative cut-off function on $\omega_{e}$ and by applying norm equivalences in finite dimensional spaces), we find an auxiliary function 
$\chi_{e} \in H_{0}^{1}\left(\omega_{e}\right) \cap C^{\infty}\left(\bar{\omega}_{e}\right)$ (which depends on the function $\left.\llbracket \nabla u_{\mathrm{DG}} \rrbracket\right|_{e}$ ) with the following properties:

$$
\left.\chi_{e}\right|_{\partial \omega_{e}}=0,\left.\quad \nabla \chi_{e}\right|_{\partial \omega_{e}}=\mathbf{0},\left.\quad \llbracket \nabla \chi_{e} \rrbracket\right|_{e}=0,
$$

as well as

$$
\left\|\llbracket \nabla u_{\mathrm{DG}} \rrbracket\right\|_{L^{2}(e)}^{2} \leq C \int_{e} \chi_{e} \llbracket \nabla u_{\mathrm{DG}} \rrbracket \mathrm{d} s,
$$

and

$$
\left\|\mathrm{h}^{-\frac{1}{2}} \nabla \chi_{e}\right\|_{L^{2}(e)}+\left\|\mathrm{h}^{-2} \chi_{e}\right\|_{L^{2}\left(\omega_{e}\right)}+\left\|\chi_{e}\right\|_{H^{2}\left(\omega_{e}\right)} \leq C\left\|\mathrm{~h}^{-\frac{3}{2}} \llbracket \nabla u_{\mathrm{DG}} \rrbracket\right\|_{L^{2}(e)} .
$$

Then, we have

$$
C\left\|\llbracket \nabla u_{\mathrm{DG}} \rrbracket\right\|_{L^{2}(e)}^{2} \leq \int_{e} \chi_{e} \llbracket \nabla u_{\mathrm{DG}} \rrbracket \mathrm{d} s=\int_{\partial K_{\sharp}} \chi_{e}\left(\nabla u_{\mathrm{DG}} \cdot \boldsymbol{n}_{K_{\sharp}}\right) \mathrm{d} s+\int_{\partial K_{\mathrm{b}}} \chi_{e}\left(\nabla u_{\mathrm{DG}} \cdot \boldsymbol{n}_{K_{\mathrm{b}}}\right) \mathrm{d} s .
$$

Applying Green's formula, we obtain

$$
\begin{aligned}
C\left\|\llbracket \nabla u_{\mathrm{DG}} \rrbracket\right\|_{L^{2}(e)}^{2} & \leq \int_{\omega_{e}} \nabla \chi_{e} \cdot \nabla_{h} u_{\mathrm{DG}} \mathrm{d} \boldsymbol{x}+\int_{\omega_{e}} \chi_{e} \Delta_{h} u_{\mathrm{DG}} \mathrm{d} \boldsymbol{x} \\
& =-\int_{\omega_{e}} \nabla \chi_{e} \cdot \nabla_{h}\left(u-u_{\mathrm{DG}}\right) \mathrm{d} \boldsymbol{x}+\int_{\omega_{e}} \chi_{e}\left(\Delta_{h} u_{\mathrm{DG}}+\delta_{h}\right) \mathrm{d} \boldsymbol{x}-\int_{\omega_{e}}\left(\delta_{h}-\delta_{\boldsymbol{x}_{0}}\right) \chi_{e} \mathrm{~d} \boldsymbol{x},
\end{aligned}
$$

where $\Delta_{h}$ signifies the elementwise Laplacian. Integrating by parts we get

$$
-\int_{\omega_{e}} \nabla \chi_{e} \cdot \nabla_{h}\left(u-u_{\mathrm{DG}}\right) \mathrm{d} \boldsymbol{x}=-\int_{e} \nabla \chi_{e} \cdot \llbracket u-u_{\mathrm{DG}} \rrbracket \mathrm{d} s+\int_{\omega_{e}}\left(u-u_{\mathrm{DG}}\right) \Delta_{h} \chi_{e} \mathrm{~d} \boldsymbol{x} .
$$

Thence,

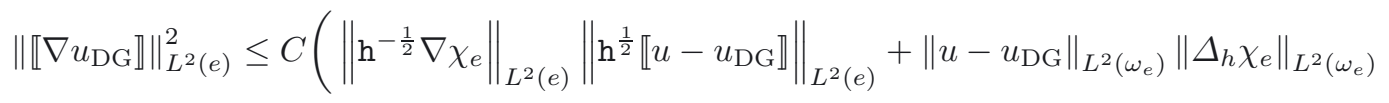

$$
\begin{aligned}
& \left.+\left\|\chi_{e}\right\|_{L^{2}\left(\omega_{e}\right)}\left\|\Delta_{h} u_{\mathrm{DG}}+\delta_{h}\right\|_{L^{2}\left(\omega_{e}\right)}+\left\|\delta_{h}-\delta_{\boldsymbol{x}_{0}}\right\|_{H^{-2}(\Omega)}\left\|\chi_{e}\right\|_{H^{2}\left(\omega_{e}\right)}\right) .
\end{aligned}
$$

Furthermore, we have

$$
\begin{aligned}
& \left\|\llbracket \nabla u_{\mathrm{DG}} \rrbracket\right\|_{L^{2}(e)}^{2} \leq C\left(\left\|\mathrm{~h}^{\frac{1}{2}} \llbracket u-u_{\mathrm{DG}} \rrbracket\right\|_{L^{2}(e)}^{2}+\left\|u-u_{\mathrm{DG}}\right\|_{L^{2}\left(\omega_{e}\right)}^{2}+\left\|\mathrm{h}^{2}\left(\Delta_{h} u_{\mathrm{DG}}+\delta_{h}\right)\right\|_{L^{2}\left(\omega_{e}\right)}^{2}\right. \\
& \left.+\left\|\delta_{h}-\delta_{\boldsymbol{x}_{0}}\right\|_{H^{-2}(\Omega)}^{2}\right)^{\frac{1}{2}} \times\left(\left\|\mathrm{h}^{-\frac{1}{2}} \nabla \chi_{e}\right\|_{L^{2}(e)}^{2}+\left\|\mathrm{h}^{-2} \chi_{e}\right\|_{L^{2}\left(\omega_{e}\right)}^{2}+\left\|\chi_{e}\right\|_{H^{2}\left(\omega_{e}\right)}^{2}\right)^{\frac{1}{2}} .
\end{aligned}
$$

Using (4.11), and recalling the previous Proposition 4.3, it follows that

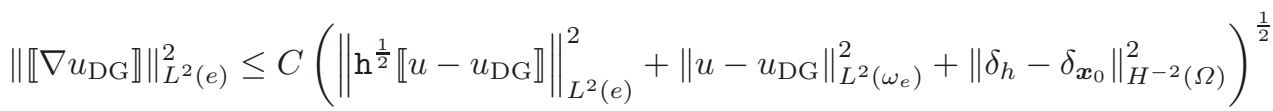

$$
\begin{aligned}
& \times\left\|\mathrm{h}^{-\frac{3}{2}} \llbracket \nabla u_{\mathrm{DG}} \rrbracket\right\|_{L^{2}(e)} .
\end{aligned}
$$

Now, noting that $\left.\mathrm{h}\right|_{\omega_{e}} \sim h_{K_{\sharp}} \sim h_{K_{\mathrm{b}}}$, completes the proof. 
Finally, we have the identity

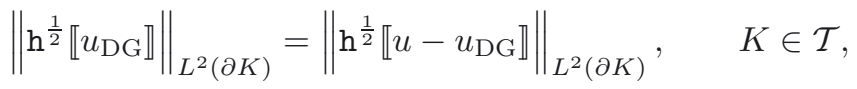

by observing again that $\left.\llbracket u \rrbracket\right|_{\mathcal{E}}=0$.

Remark 4.6. The term $\left\|\delta_{h}-\delta_{x_{0}}\right\|_{H^{-2}(\Omega)}$ appearing in the lower error estimates above takes the role of a data approximation term. We note that

$$
\begin{aligned}
\int_{\Omega}\left(\delta_{\boldsymbol{x}_{0}}-\delta_{h}\right) \psi \mathrm{d} \boldsymbol{x} & =\left(\psi-\psi_{h}\right)\left(\boldsymbol{x}_{0}\right)-\int_{\Omega} \delta_{h}\left(\psi-\psi_{h}\right) \\
& \leq \sup _{\boldsymbol{x} \in K_{0}}\left|\left(\psi-\psi_{h}\right)(\boldsymbol{x})\right|+\left\|\delta_{h}\right\|_{L^{2}\left(K_{0}\right)}\left\|\psi-\psi_{h}\right\|_{L^{2}\left(K_{0}\right)}
\end{aligned}
$$

for any $\psi \in H^{2}(\Omega)$ and any $\psi_{h} \in V_{\mathrm{DG}}(\mathcal{T})$. Let us choose $\psi_{h} \in \mathbb{S}_{1}(K), K \in \mathcal{T}$, to be an interpolant that satisfies the standard approximation estimate

$$
h_{K}^{-2}\left\|\psi-\psi_{h}\right\|_{L^{2}(K)}^{2}+\left\|\nabla\left(\psi-\psi_{h}\right)\right\|_{L^{2}(K)}^{2} \leq C h_{K}^{2}\|\psi\|_{H^{2}(K)}^{2}, \quad K \in \mathcal{T} .
$$

Evidently, since $\nabla^{2} \psi_{h} \equiv 0$ on each element, we additionally have that

$$
\left\|\nabla^{2}\left(\psi-\psi_{h}\right)\right\|_{L^{2}(K)} \leq C\|\psi\|_{H^{2}(K)},
$$

where the constant $C>0$ is independent of $\boldsymbol{h}$. Furthermore, due to the continuous Sobolev embedding $H^{2}(\Omega) \hookrightarrow$ $L^{\infty}(\Omega)$ (see, e.g., [1]), and by using a scaling argument, we conclude that

$$
\sup _{\boldsymbol{x} \in K}\left|\left(\psi-\psi_{h}\right)(\boldsymbol{x})\right| \leq C h_{K}\|\psi\|_{H^{2}(K)} .
$$

Therefore, using the above bounds, together with (3.5), we obtain

$$
\int_{\Omega}\left(\delta_{\boldsymbol{x}_{0}}-\delta_{h}\right) \psi \mathrm{d} \boldsymbol{x} \leq C h_{K_{0}}\|\psi\|_{H^{2}\left(K_{0}\right)}
$$

for a constant $C>0$ independent of $\boldsymbol{h}$. Therefore,

$$
\left\|\delta_{\boldsymbol{x}_{0}}-\delta_{h}\right\|_{H^{-2}(\Omega)}=\sup _{\psi \in H^{2}(\Omega), \psi \neq \equiv 0} \frac{\int_{\Omega}\left(\delta_{\boldsymbol{x}_{0}}-\delta_{h}\right) \psi \mathrm{d} \boldsymbol{x}}{\|\psi\|_{H^{2}(\Omega)}} \leq C h_{K_{0}} .
$$

\subsection{Application to the SIPG method}

We will now apply Theorem 4.1 to the SIPG method (2.4). More precisely, the quantity $\Upsilon\left(u_{\mathrm{DG}}\right)$ from $(4.6)$ will be defined explicitly. To this end, we start by noticing that the numerical fluxes in the SIPG form $a_{\mathrm{DG}}(\cdot, \cdot)$ from (2.4) satisfy

$$
\mathcal{F}\left(u_{\mathrm{DG}}, \psi_{h}\right)=-\int_{\mathcal{E}}\left\langle\left\langle\nabla_{h} u_{\mathrm{DG}}\right\rangle\right\rangle \cdot \llbracket \psi_{h} \rrbracket \mathrm{d} s-\int_{\mathcal{E}} \llbracket u_{\mathrm{DG}} \rrbracket \cdot\left\langle\left\langle\nabla_{h} \psi_{h}\right\rangle\right\rangle \mathrm{d} s+\gamma \int_{\mathcal{E}} \mathrm{h}^{-1} \llbracket u_{\mathrm{DG}} \rrbracket \cdot \llbracket \psi_{h} \rrbracket \mathrm{d} s
$$

for any $\psi_{h} \in V_{\mathrm{DG}}(\mathcal{T})$. Consequently, the residual $\mathcal{R}$ from (4.5) satisfies

$$
\mathcal{R}\left[u_{\mathrm{DG}}, \psi\right]\left(\psi_{h}\right)=\int_{\mathcal{E}} \llbracket u_{\mathrm{DG}} \rrbracket \cdot\left\langle\left\langle\nabla_{h}\left(\psi-\psi_{h}\right)\right\rangle\right\rangle \mathrm{d} s+\gamma \int_{\mathcal{E}} \mathrm{h}^{-1} \llbracket u_{\mathrm{DG}} \rrbracket \cdot \llbracket \psi_{h} \rrbracket \mathrm{d} s .
$$


Using that $\psi \in H_{0}^{1}(\Omega)$, we notice that $\llbracket \psi \rrbracket=0$ on $\mathcal{E}$. Therefore, we obtain

$$
\begin{aligned}
& \left|\mathcal{R}\left[u_{\mathrm{DG}}, \psi\right]\left(\psi_{h}\right)\right| \leq\left|\int_{\mathcal{E}} \llbracket u_{\mathrm{DG}} \rrbracket \cdot\left\langle\left\langle\nabla_{h}\left(\psi-\psi_{h}\right)\right\rangle\right\rangle \mathrm{d} s\right|+\left|\gamma \int_{\mathcal{E}} \mathrm{h}^{-1} \llbracket u_{\mathrm{DG}} \rrbracket \cdot \llbracket \psi-\psi_{h} \rrbracket \mathrm{d} s\right|
\end{aligned}
$$

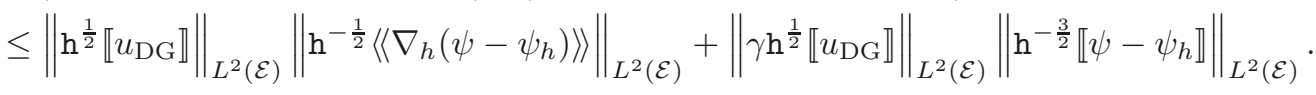

Employing the Cauchy-Schwarz inequality, this implies (4.6), with

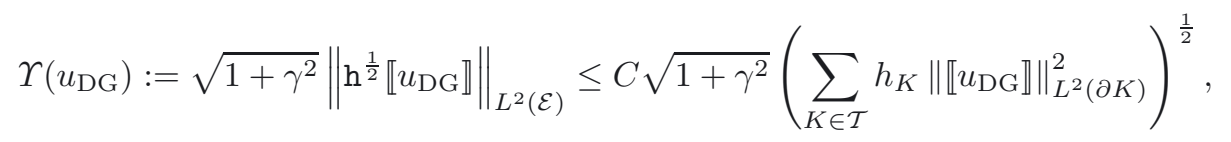

and

$$
\begin{aligned}
\left\|\psi-\psi_{h}\right\|_{h}^{2} & :=\| \mathrm{h}^{-\frac{1}{2}}\left\langle\left\langle\nabla_{h}\left(\psi-\psi_{h}\right)\right\rangle\left\|_{L^{2}(\mathcal{E})}^{2}+\right\| \mathrm{h}^{-\frac{3}{2}} \llbracket \psi-\psi_{h} \rrbracket \|_{L^{2}(\mathcal{E})}^{2}\right. \\
& \leq C \sum_{K \in \mathcal{T}}\left(h_{K}^{-3}\left\|\psi-\psi_{h}\right\|_{L^{2}(\partial K)}^{2}+h_{K}^{-1}\left\|\nabla\left(\psi-\psi_{h}\right)\right\|_{L^{2}(\partial K)}^{2}\right) .
\end{aligned}
$$

Applying the trace inequality, with scaling, yields

$$
\left\|\psi-\psi_{h}\right\|_{h}^{2} \leq C \sum_{K \in \mathcal{T}}\left(h_{K}^{-4}\left\|\psi-\psi_{h}\right\|_{L^{2}(K)}^{2}+h_{K}^{-2}\left\|\nabla\left(\psi-\psi_{h}\right)\right\|_{L^{2}(K)}^{2}+\left\|\nabla^{2}\left(\psi-\psi_{h}\right)\right\|_{L^{2}(K)}^{2}\right) .
$$

We choose $\psi_{h} \in V_{\mathrm{DG}}(\mathcal{T})$ to be an interpolant of $\psi$ that fulfils the bounds (4.13)-(4.15); this then implies that (4.7) holds.

Thus, employing Theorem 4.1 and recalling (4.16), we deduce the following result.

Theorem 4.7. The SIPG method (2.4) for the numerical approximation of (1.1)-(1.2) satisfies the a posteriori error estimate

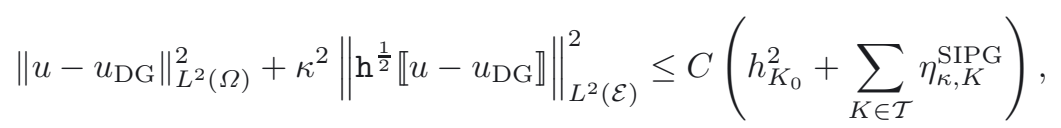

where

$$
\eta_{\kappa, K}^{\mathrm{SIPG}}:=h_{K}^{4}\left\|\Delta u_{\mathrm{DG}}+\delta_{h}\right\|_{L^{2}(K)}^{2}+h_{K}^{3}\left\|\llbracket \nabla_{h} u_{\mathrm{DG}} \rrbracket\right\|_{L^{2}(\partial K \backslash \partial \Omega)}^{2}+\left(1+\gamma^{2}+\kappa^{2}\right) h_{K}\left\|\llbracket u_{\mathrm{DG}} \rrbracket\right\|_{L^{2}(\partial K)}^{2},
$$

for any $K \in \mathcal{T}$, and any constant $\kappa>0$. Here, $C>0$ is a constant independent of $\boldsymbol{h}, u_{\mathrm{DG}}$, $\gamma$, and of $\kappa$.

Remark 4.8. Local lower a posteriori error estimates for the SIPG scheme are given by the generally valid estimates from Section 4.2. Evidently, a sensible choice of $\kappa$ is given by $\kappa \sim \gamma$. This will ensure the equivalence of the terms

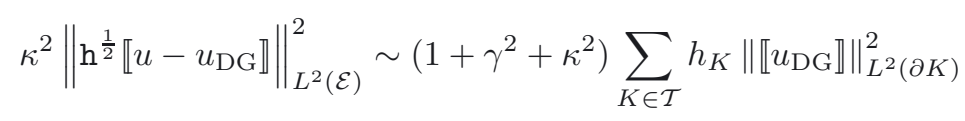

appearing on the left and right-hand side of the a posteriori error estimate (4.17), respectively; $c f$. (4.12).

Remark 4.9. We note that the convexity of $\Omega$ is not essential in the a posteriori error analysis above. In the non-convex case, however, the presence of possible corner singularities in the solution $\psi$ of (4.1)-(4.2) implies that $\psi \in W^{2, p}(\Omega)$ for some $p<2$ rather than $\psi \in H^{2}(\Omega)$; see, e.g., [11]. Consequently, a refined analysis based on $L^{p}$ spaces is required. This can again be done along the lines of [3]; cf. also [19]. 


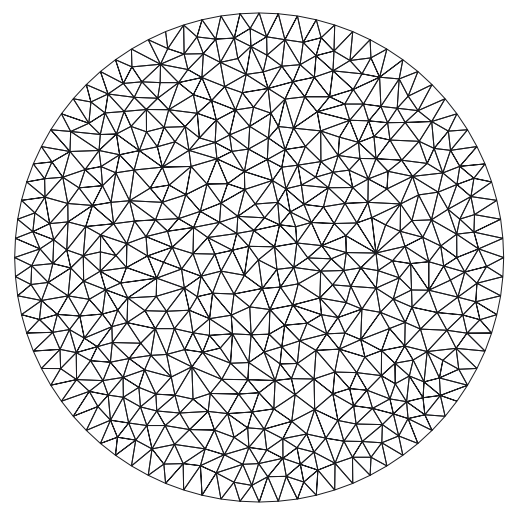

FiguRE 1. Initial mesh, consisting of 988 elements.

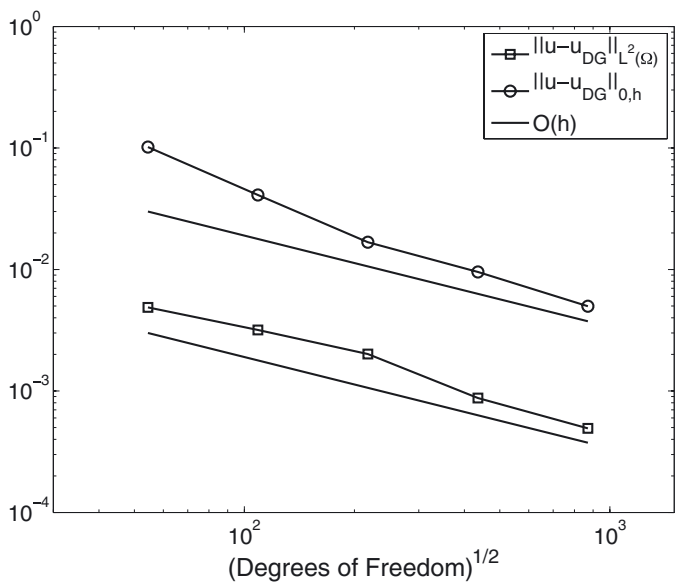

(a)

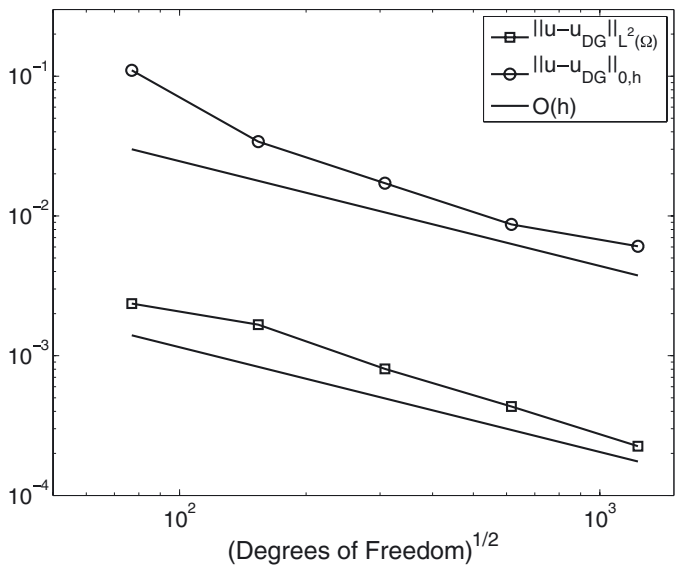

(b)

FigURE 2. Convergence of $\left\|u-u_{\mathrm{DG}}\right\|_{L^{2}(\Omega)}$ and $\left\|u-u_{\mathrm{DG}}\right\|_{0, h}$ on quasi-uniform triangular meshes with (a) $\ell=1$; (b) $\ell=2$.

\section{NUMERICAL EXAMPLES}

We consider the case when the computational domain $\Omega$ is the unit disc, i.e., $\Omega=\left\{\boldsymbol{x} \in \mathbb{R}^{2}:|\boldsymbol{x}|<1\right\}$. Setting $\boldsymbol{x}_{0}=\mathbf{0}$, the analytical solution to (1.1)-(1.2) is the fundamental solution of the Laplace equation; namely,

$$
u(\boldsymbol{x})=-\frac{1}{2 \pi} \ln |\boldsymbol{x}| .
$$

Our numerical experiments are based on the SIPG method (2.4); here, we choose $\gamma=\kappa=10$. Firstly, we investigate the asymptotic convergence of the SIPG method on a sequence of successively finer quasi-uniform unstructured triangular meshes for $\ell=1,2$. The initial mesh consists of 988 elements; $c f$. Figure 1 . Here, curved elements have not been employed on the boundary of the computational domain; however, when elements which lie on the boundary are refined, then new nodal points are automatically placed on the circular boundary of the domain. In Figure 2 we present a comparison of the $L^{2}(\Omega)$-norm, as well as the extended $L^{2}(\Omega)$-norm defined in Remark 4.2, of the error $u-u_{\mathrm{DG}}$ for $\ell=1,2$, as the initial mesh is uniformly refined. Here, we observe that 


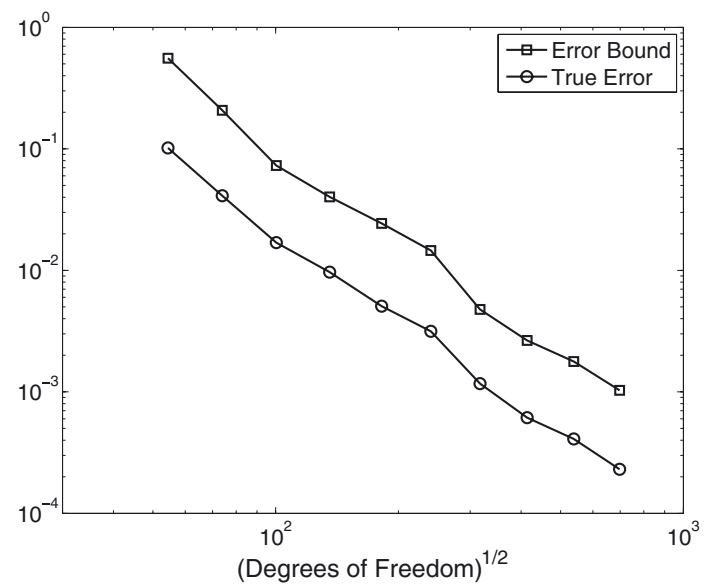

(a)

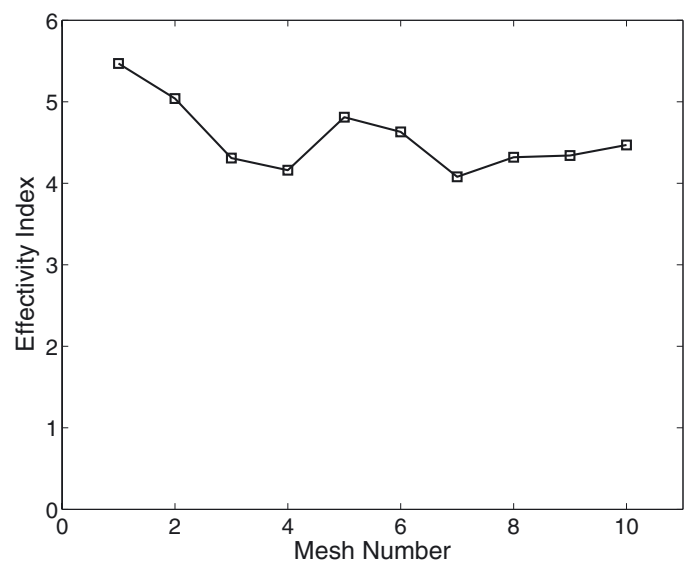

(b)

Figure 3. (a) Comparison of the actual and estimated extended $L^{2}(\Omega)$-norm of the error with respect to the number of degrees of freedom; (b) effectivity indices.

(asymptotically) $\left\|u-u_{\mathrm{DG}}\right\|_{L^{2}(\Omega)}$ converges to zero at the rate $\mathcal{O}(h)$ as $h$ tends to zero, $c f$. Theorem 3.1. Similar behavior of the norm $\left\|u-u_{\mathrm{DG}}\right\|_{0, h}$ is also observed asymptotically.

Secondly, we now investigate the performance of the a posteriori error estimate derived in Theorem 4.7 within an automatic $h$-version adaptive refinement procedure which is based on 1-irregular triangular elements, with $\ell=1$. The $h$-adaptive meshes are constructed by marking the elements for refinement/derefinement according to the size of the local error indicators defined on the right-hand side of (4.17); this is done by employing the fixed fraction strategy. This involves choosing two numbers $\varphi_{\text {ref }}$ and $\varphi_{\text {deref }}$ in the interval $(0,100)$ with $\varphi_{\text {deref }}+\varphi_{\text {ref }}<100$, ordering the local refinement indicators, according to their size, and then refining those elements $\kappa$ which correspond to $\varphi_{\text {ref }} \%$ of the largest entries in the ordered sequence, and derefining those elements $\kappa$ which correspond to the $\varphi_{\text {deref }} \%$ of the smallest entries in this ordered sequence; further variations on this strategy, with dynamically varying $\varphi_{\text {ref }}$ and $\varphi_{\text {deref }}$, are also possible. For the purposes of this section, we set refinement and derefinement fractions $\varphi_{\text {ref }}$ and $\varphi_{\text {deref }}$, respectively, equal to $25 \%$ and $10 \%$, respectively. In two-dimensions, this leads to roughly a doubling of the number of degrees of freedom in the underlying finite element space at each refinement step.

The initial starting mesh for adaptive refinement is the same one depicted in Figure 1. In Figure 3a we show the history of the actual and estimated extended $L^{2}(\Omega)$-norm of the error on each of the meshes generated based on employing $h$-adaptive mesh refinement. Here, we observe that the a posteriori bound over-estimates the true error by a consistent factor. Indeed, the effectivity index tends to a value of around 4 as the mesh is adaptively refined, $c f$. Figure 3b. In Figure 4 we plot the meshes overlayed onto the corresponding computed DG solution after 0 (initial mesh), 2, 4, 6, 8, and 9 adaptive refinement steps have been undertaken. Here, we observe that the mesh has been significantly refined in the vicinity of the origin of the computational domain, where the delta-source term is centered, as expected.

\section{Conclusions}

In this article we have developed both the a priori and a posteriori error analysis of a general class of DG finite element methods for the numerical approximation of linear second-order elliptic partial differential equations with Dirac delta right-hand side. In particular, the a priori bound indicates that the $L^{2}$-norm of the discretization error converges to zero at the rate $\mathcal{O}(h)$ as the mesh size $h$ tends to zero. Secondly, computable 


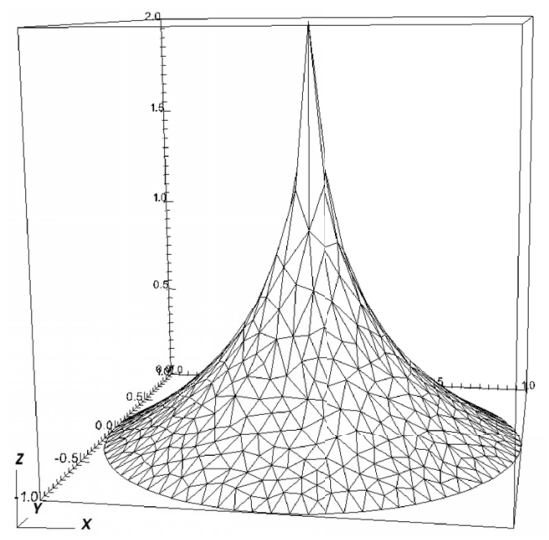

(a)

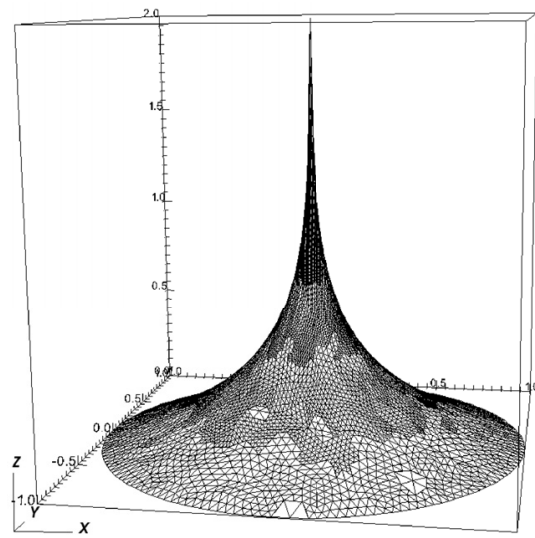

(c)

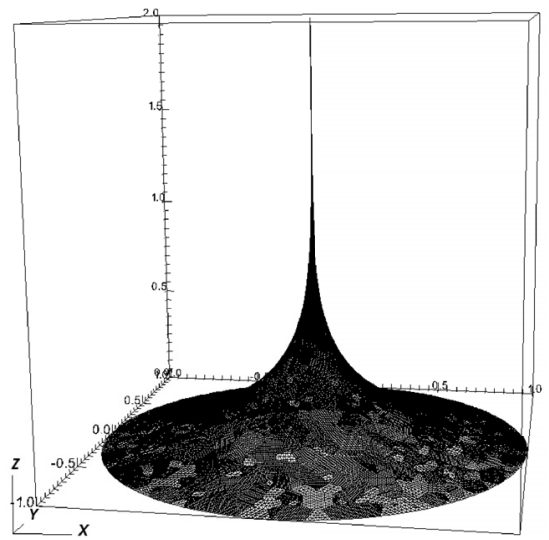

(e)

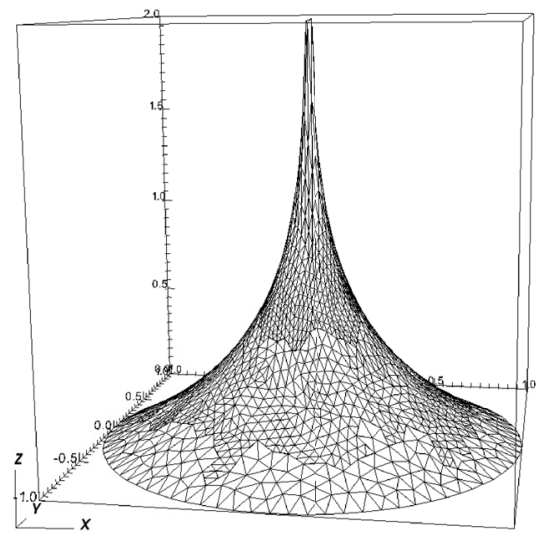

(b)

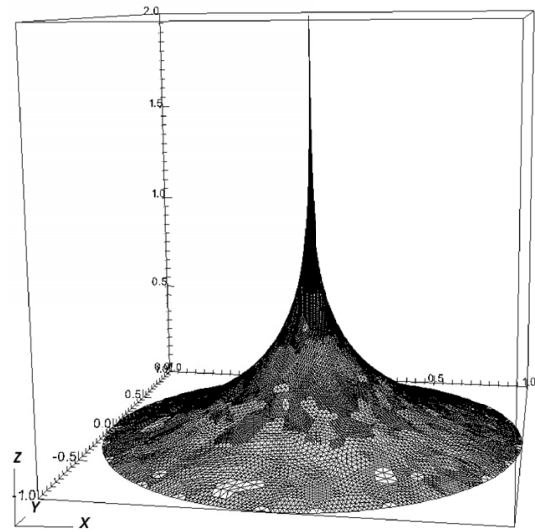

(d)

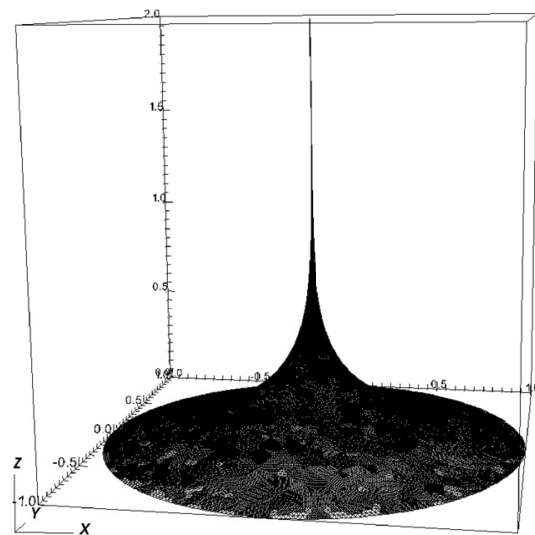

(f)

FiguRE 4. DG solution and mesh. (a) Initial mesh, with 988 elements; (b) 2 adaptive refinements, with 3358 elements; (c) 4 adaptive refinements, with 11032 elements; (d) 6 adaptive refinements, with 33538 elements; (e) 8 adaptive refinements, with 96646 elements; (f) 9 adaptive refinements, with 162259 elements. 
residual-based a posteriori error indicators have been derived when the error is measured in terms of an extended $L^{2}$-norm; the use of this norm facilitates the derivation of local lower bounds. These theoretical results have been confirmed numerically; in particular, the a posteriori error bound has been employed within an automatic adaptive mesh refinement algorithm.

\section{REFERENCES}

[1] R.A. Adams and J.J.F. Fournier, Sobolev Spaces. Elsevier (2003).

[2] T. Apel, O. Benedix, D. Sirch and B. Vexler, A priori mesh grading for an elliptic problem with Dirac right-hand side. SIAM J. Numer. Anal. 49 (2011) 992-1005.

[3] R. Araya, E. Behrens and R. Rodríguez. A posteriori error estimates for elliptic problems with Dirac delta source terms. Numer. Math. 105 (2006) 193-216.

[4] D.N. Arnold, An interior penalty finite element method with discontinuous elements. SIAM J. Numer. Anal. 19 (1982) $742-760$.

[5] D.N. Arnold, F. Brezzi, B. Cockburn and L.D. Marini, Unified analysis of discontinuous Galerkin methods for elliptic problems. SIAM J. Numer. Anal. 39 (2001) 1749-1779.

[6] R. Becker and R. Rannacher, An optimal control approach to a-posteriori error estimation in finite element methods, edited by A. Iserles. Cambridge University Press. Acta Numerica (2001) 1-102.

[7] E. Casas, $L^{2}$ estimates for the finite element method for the Dirichlet problem with singular data. Numer. Math. 47 (1985) $627-632$.

[8] M. Dauge, Elliptic Boundary Value Problems on Corner Domains. Lect. Notes Math. 1341 (1988).

[9] J. Douglas and T. Dupont, Interior penalty procedures for elliptic and parabolic Galerkin methods, in Computing methods in applied sciences (Second Internat. Sympos., Versailles, 1975). Lect. Notes Phys. 58 (1976) 207-216.

[10] K. Eriksson, D. Estep, P. Hansbo and C. Johnson, Introduction to adaptive methods for differential equations, edited by A. Iserles. Cambridge University Press. Acta Numerica (1995) 105-158.

[11] P. Grisvard, Elliptic Problems in Nonsmooth Domains. Pitman (1985).

[12] P. Houston and E. Süli, Adaptive finite element approximation of hyperbolic problems, in Error Estimation and Adaptive Discretization Methods in Computational Fluid Dynamics, edited by T. Barth and H. Deconinck. Lect. Notes Comput. Sci. Eng. 25 (2002).

[13] P. Houston and T.P. Wihler, Second-order elliptic PDE with discontinuous boundary data. IMA J. Numer. Anal. 32 (2012) $48-74$.

[14] V. John, A posteriori $L^{2}$-error estimates for the nonconforming $P_{1} / P_{0}$-finite element discretization of the Stokes equations. J. Comput. Appl. Math. 96 (1998) 99-116.

[15] B. Rivière, Discontinuous Galerkin Methods for Solving Elliptic and Parabolic Equations, Theory and Implementation, in Front. Appl. Math. SIAM (2008).

[16] B. Rivière, M.F. Wheeler and V. Girault, A priori error estimates for finite element methods based on discontinuous approximation spaces for elliptic problems. SIAM J. Numer. Anal. 39 (2001) 902-931 (electronic).

[17] R. Scott, Finite element convergence for singular data. Numer. Math. 21 (1973/1974) 317-327.

[18] M.F. Wheeler, An elliptic collocation finite element method with interior penalties. SIAM J. Numer. Anal. 15 (1978) $152-161$.

[19] T.P. Wihler and B. Rivière, Discontinuous Galerkin methods for second-order elliptic PDE with low-regularity solutions. J. Sci. Comput. 46 (2011) 151-165. 